\title{
Contrast enhancement of carotid adventitial vasa vasorum as a biomarker of radiation-induced atherosclerosis
}

${ }^{1}$ Dorothy M. Gujral, ${ }^{2}$ Wing Keung Cheung, ${ }^{3}$ Benoy N. Shah, ${ }^{3}$ Navtej S. Chahal, ${ }^{3}$ Sanjeev Bhattacharyya, ${ }^{4}$ James Hooper, ${ }^{3}$ Roxy Senior, ${ }^{2}$ Meng-Xing Tang, ${ }^{1}$ Kevin J. Harrington, ${ }^{\star 1}$ Christopher M. Nutting

${ }^{1}$ Head and Neck Unit, The Royal Marsden Hospital, 203 Fulham Road, London, SW3 6JJ, UK

${ }^{2}$ Department of Bioengineering, Imperial College, Exhibition Road, London, SW7 2AZ ${ }^{3}$ Department of Echocardiography, Royal Brompton Hospital, Sydney Street, London, SW3 $6 N P$

${ }^{4}$ Department of Biochemistry, Royal Brompton Hospital, Sydney Street, London, SW3 6NP

*Corresponding author: Prof Christopher Nutting, Head and Neck Unit, Royal Marsden Hospital, Fulham Rd, London, SW3 6JJ, UK

E-mail: Christopher.Nutting@rmh.nhs.uk, Telephone: 0044207808 2586; Fax: 0044207 8082235

Running title: CEUS in irradiated carotid arteries

Keywords: biomarker, carotid, radiotherapy, atherosclerosis, neovascularisation, contrastenhanced ultrasound

Word Count: 


\section{ABSTRACT}

Purpose

Abnormal proliferation of adventitial vasa vasorum (vv) occurs early at sites of atherosclerosis and is thought to be an early biomarker of vascular damage. Contrastenhanced ultrasound (CEUS) can detect this process. Its usefulness in irradiated arteries as a measure of accelerated atherosclerosis is unknown. This study investigates contrast intensity in carotid adventitia as an early marker of radiation-induced damage in head and neck cancer (HNC) patients.

\section{Materials/Methods}

Patients with HNC treated with a wedged-pair and matched neck technique or hemi-neck radiotherapy (RT) (unirradiated side as control) at least 2 years previously were included. Patients had been prescribed a dose of at least 50 Gy to the neck. CEUS was performed on both carotid arteries and a region of interest was selected in the adventitia of the far wall of both left and right distal common carotid arteries. Novel quantification software was used to compare the average intensity per pixel between irradiated and unirradiated arteries.

Results

48 patients (34 males) with median age of 59.2 yrs (interquartile range (IQR) $49.2-64.2$ ) were included. The mean maximum point dose to the irradiated artery was 61.2 Gy (IQR $52.6-61.8$ ) and 1.1 Gy (IQR $1.0-1.8 \mathrm{~Gy})$ to the unirradiated side. The median interval from RT was 59.4 months (IQR 41 - 88.7). There was a significant difference in the mean (SD) contrast intensity per pixel on the irradiated side $(1.1(0.4))$ versus $0.96(0.34)$ on the unirradiated side $(p=0.01)$. After attenuation correction, the difference in mean contrast intensity per pixel was still significant $(1.4(0.58)$ versus $1.2(0.47)(p=0.02)$. Previous surgery or chemotherapy had no effect on the difference in contrast intensity between the 2 sides of the neck. Mean intensity per pixel did not correlate to traditional risk prediction models (carotid intima-medial thickness, QSTROKE score). 
Conclusions

Proliferation of $v v$ is demonstrated by increased contrast intensity in irradiated carotid arteries. This may be a useful, independent biomarker of radiation-induced carotid atherosclerosis when used as a tool to quantify neovascularisation.

Keywords: biomarker, carotid, radiotherapy, atherosclerosis, neovascularisation, contrastenhanced ultrasound 


\section{INTRODUCTION}

Vasa vasorum (vv) are a plexus of microvessels located in the adventitia of most medium and large arteries (including the aorta, coronary, femoral and carotid arteries). Their function is primarily related to the nutritional needs of veins and arteries. Researchers have demonstrated an increase in the density of adventitial vv before the development of atherosclerotic lesions in porcine coronary arteries (1). Herrmann et al (2) further reported that increase in the density of adventitial $\mathrm{v}$ occurs before the onset of endothelial dysfunction (one of the first functional alterations in atherosclerosis). Studies in humans have identified plaque neovascularization as a key feature of vulnerable plaques, i.e. those plaques at risk of rupture $(3,4)$. Furthermore, abnormal proliferation of human adventitial vv occurs early at sites of atherosclerosis and is thought to be a precursor to atherosclerosis and an early biomarker of vascular damage $(4,5)$.

Contrast-enhanced ultrasound (CEUS) imaging of the carotid arteries enables the acquisition of real-time carotid images with enhancement of the arterial lumen and plaque morphology, improved resolution of CIMT, and, importantly, direct visualization of the adventitial vv and plaque neovascularization (6-8). This is an attractive diagnostic tool due to its non-ionizing nature and real-time imaging with good spatial resolution, relatively low cost and high accessibility. Contrast agents used in CEUS are microbubbles. These are small (2 to $8 \mu \mathrm{m}$ ) acoustically-active particles composed of a gaseous core of perfluorocarbons surrounded by a biocompatible shell composed of a combination of lipids, albumin, or polymers (6). When exposed to an ultrasound beam, these particles expand and contract, creating backscatter and, consequently, enabling them to act as echo enhancers. Techniques with multi-pulse sequence transmission and signal processing have been widely used to improve the imaging specificity of microbubbles and have made it possible to image and quantify neovascularization in plaques and in adventitial vv (4, 9-13). However, current quantification of contrast enhancement is limited by spatially 
heterogeneous and patient-specific attenuation $(14,15)$. Accounting for these variables will allow for improved quantification of signal intensity from CEUS as a measure of adventitial neovascularization. Some studies have already attempted to correct for attenuation $(14,16$, 17).

The aim of this study was to examine the contrast intensity in the adventitial vv of irradiated arteries and compare this to unirradiated arteries. We hypothesized that radiation-induced injury results in an inflammatory response in the endothelium and consequent proliferation of adventitial vv, which may be measured quantitatively using CEUS. The effect of surgery, as well as RT, to the neck was also studied and the difference in contrast intensity between irradiated and unirradiated carotid arteries was correlated to the interval from RT to see if contrast intensity increases early after RT. In addition, this study aimed to see if there is any correlation between contrast intensity and validated markers for risk prediction (carotid intima-medial thickness (CIMT) and QSTROKE score).

\section{MATERIALS AND METHODS}

Head and neck cancer patients treated with RT (minimum dose of $50 \mathrm{~Gy}$ ) to one side of the neck prior to December 2009 were included in the study. Risk factor determination was performed as follows - medical history, blood pressure, body mass index, blood glucose, and lipid profile. Ethical approval was obtained from a regional Research Ethics Committee and the protocol for the study was reviewed by the Royal Marsden Committee for Clinical Research and registered on clinicaltrials.gov (NCT02060643).

Study assessments

Head and neck cancer patients treated prior to December 2009 were prospectively identified via the Royal Marsden Hospital head and neck RT database. Written informed consent was obtained from each participant and medication history and co-morbidities were recorded. 
Blood pressure, height and weight (to determine body mass index) were measured and electrocardiogram (ECG) was performed. Blood samples were taken for haemoglobin, plasma glucose, lipid profile, renal and liver profile.

\section{Definitions}

The following definitions were used in this study:

- Hypertension - systolic blood pressure (SBP) $\geq 140 \mathrm{mmHg}$ and/or diastolic blood pressure $(\mathrm{DBP}) \geq 90 \mathrm{mmHg}$

- Diabetic - random serum glucose $\geq 11.1 \mathrm{mmol} / \mathrm{L}$, a glycosylated haemoglobin $\mathrm{A} 1 \mathrm{c}$ $(\mathrm{HbA} 1 \mathrm{c}) \geq 5.8 \%$ or current use of glucose-lowering agents or insulin

- Hyperlipidaemia - fasting serum low density lipoprotein $(L D L) \geq 2.6 \mathrm{mmol} / \mathrm{L}$, high density lipoprotein $(\mathrm{HDL})<2.3 \mathrm{mmol} / \mathrm{L}$ or triglycerides $(\mathrm{TG}) \geq 2.3 \mathrm{mmol} / \mathrm{L}$, or current use of cholesterol-lowering agents. Normal ranges: HDL cholesterol $-1.0-2.3 \mathrm{mmol} / \mathrm{L}$; LDL cholesterol - $<2.6 \mathrm{mmol} / \mathrm{L}$; TG (fasting) - $<2.3 \mathrm{mmol} / \mathrm{L}$ (ideal $=<1.5 \mathrm{mmol} / \mathrm{L}$ ); Total cholesterol $-3.6-8.0 \mathrm{mmol} / \mathrm{L}$ (non-fasting) (ideal $=<5.0 \mathrm{mmol} / \mathrm{L}$ (fasting)), Total cholesterol/HDL ratio $<5$

- Smoking history - smoker (pack years) or non-smoker/ex-smoker > 10 years

- Normal BMI - $18.5-24.9 \mathrm{~kg} / \mathrm{m}^{2}$

- Overweight - $25.0-29.9 \mathrm{~kg} / \mathrm{m}^{2}$

- Obese $-\geq 30 \mathrm{~kg} / \mathrm{m}^{2}$

- Atrial fibrillation - standard ECG changes

- Stroke - sudden onset of a neurological deficit persisting for $\geq 24$ hours

- Transient ischaemic attack (TIA) - focal neurological symptoms lasting $<24$ hours

Ultrasound studies

CEUS image sequences were acquired on both sides of the neck with a clinical scanner (GE Vivid7 with a $9 \mathrm{MHz}$ broadband linear array transducer) using a contrast-specific imaging 
pre-set. Contrast-enhanced ultrasound video loops were taken using a commercially available ultrasound contrast agent, SonoVue ${ }^{\mathrm{TM}}$ (Bracco, Milan) given as an intravenous infusion via a peripheral vein at a rate of $1.2 \mathrm{~mL} / \mathrm{min}$. The infusion was delivered over a total of 5-7 minutes. Imaging was performed in real-time prior to the arrival of and following the saturation of the carotid artery with SonoVue.

\section{CEUS quantification}

An attenuation correction and image normalization algorithm for CEUS carotid artery images was utilized and has been previously described (18) in order to reduce the effects of any non-uniform transducer-skin contact and heterogeneity in tissue attenuation on quantification. Analysis of CEUS video sequences was performed offline using software developed using Matlab (The Mathworks Inc., Natick, MA, USA). Regions of interest (ROIs) were selected manually, one to segment the lumen and the others to include regions in adventitia where quantification was required (18). The motion of the lumen and adventitial ROls in the video sequence was tracked and corrected by employing a piece-wise block matching algorithm (19). As a result of motion correction, all images in the sequence were aligned to the first image. As the size of the ROls differed for each video sequence, the average intensity per pixel was calculated by dividing the total intensity for the ROI by the ROI size (which was calculated in pixels). This was undertaken for video sequences obtained before (subtraction) and after contrast infusion.

\section{CIMT}

B-mode imaging using a $9 \mathrm{MHz}$ linear array transducer prior to infusion of contrast agent was utilized to visualize the CIMT. The common carotid (CCA) and the proximal portion of the internal carotid (ICA) and external carotid arteries (ECA) on both sides of the neck were examined with the patient supine on an examination couch. Four-beat video loops (longaxis and short-axis) were stored for offline analysis. 
CIMT was determined offline using semi-automated edge-detection software. Measurements were blinded as clinical details were not available at the time of analysis. Magnified still images were used for analysis and measurements were taken from the far wall of the CCA away from any atherosclerotic plaque, $1 \mathrm{~cm}$ proximal to the bulb. Mean CIMT was recorded and the average of 3 readings was taken for each measurement.

\section{QSTROKE score}

The QSTROKE score was specifically designed to aid general practitioners in predicting a patient's risk of developing a stroke and is based on the presence of atherosclerotic risk factors. A 10 -year risk of cardiovascular disease of $20 \%$ or greater is considered high risk, and $<10 \%$ is considered low risk (20). The Q-STROKE (2014) score was calculated for each patient using an online calculator (www.qstroke.org). Details of each patient's age, sex, race, co-morbidities, medication, systolic blood pressure, body mass index and cholesterol to high-density lipoprotein $(\mathrm{HDL})$ ratio were inputted into the calculator to determine a percentage 10-year risk.

Statistical analysis

Patient characteristics were summarised using descriptive statistics. Quantitative variables were expressed as means (+/- standard deviations) and medians (including ranges). Qualitative variables were expressed as frequencies and percentages.

The mean contrast intensity (before and after attenuation correction) in the irradiated adventitia versus the mean contrast intensity in the unirradiated adventitia was compared using a paired t-test. The effect of interval from RT, chemotherapy and surgery on contrast intensity was investigated using multiple linear regression. Correlation of contrast intensity to CIMT, QSTROKE and serum biomarkers was undertaken using Spearman's rank 
correlation. Contrast intensity in irradiated and unirradiated arteries before contrast infusion was compared using a paired t-test.

All statistical tests were considered two-tailed with significant difference at the $p<0.05$ level for statistical tests performed to test a single hypothesis. The Bonferroni correction was applied for the effect of surgery and chemotherapy on contrast intensity and the $\alpha$ was set at 0.025. When the contrast intensity was correlated to the time from RT and CIMT, the Bonferroni correction was applied and the $\alpha$ was set at 0.005 . This was done as a number of tests were run to test a single hypothesis. Statistical analyses were carried out using Stats Direct Medical Statistics and Graphpad Prism 6 statistical packages.

\section{RESULTS}

CEUS images for 48 patients were available for analysis. One patient declined intravenous contrast due to poor venous access and one patient had significant motion during the study due to coughing and, therefore, the algorithm was unable to track the vessel adequately. Patient characteristics are presented in Table 1. The mean maximum dose to the irradiated artery was 61.2 Gy (IQR $52.6-61.8)$ and 1.1 Gy (IQR $1.0-1.8 \mathrm{~Gy})$ to the unirradiated carotid artery. The median interval from RT was 59.4 months (IQR $41-88.7$ ).

\section{Contrast intensity}

There was a significant difference in the mean (SD) contrast intensity per pixel on the irradiated side $(1.1(0.4))$ compared to $0.96(0.34)$ on the unirradiated side $(p=0.01)$. After attenuation correction, the difference in mean contrast intensity per pixel was still significant (1.4 (0.58) versus $1.2(0.47)(p=0.02))$ (Figure $1 a+b)$.

We compared the contrast intensity before contrast (subtraction images) between the irradiated and unirradiated side. There was no difference between the signal intensity 
before contrast infusion in irradiated (mean $0.55(S D=0.18)$ ) versus unirradiated (mean $0.51(S D=0.18)(p=0.12)$ arteries, suggesting that there was no significant difference in tissue homogeneity between irradiated and unirradiated arteries before contrast infusion.

Effect of surgery and chemotherapy

There was no difference in contrast intensity in patients who had a neck dissection compared to those who had not. The same was true for patients who had received chemotherapy or not, suggesting that the difference seen between the irradiated and unirradiated side was predominantly due to RT. These results are shown in table 2 .

Correlation to validated markers for stroke risk

CIMT

As ROls were selected on the far wall of the distal CCA, we compared the difference in mean distal CCA CIMT between irradiated - unirradiated arteries to the difference in contrast intensity between irradiated - unirradiated arteries (Figure 2). There was no significant correlation between CIMT and contrast intensity either before or after attenuation correction.

\section{QSTROKE score}

There was no correlation between contrast intensity and QSTROKE score for both the irradiated and unirradiated side of the neck. The correlation coefficient $(r)$ of uncorrected contrast intensity to QSTROKE for the irradiated neck was $-0.06(p=0.68)$; corrected contrast intensity, $r=0.07(p=0.64)$. For the unirradiated neck, the correlation coefficient of uncorrected contrast intensity to QSTROKE was $-0.15(p=0.31)$; for corrected contrast intensity, $r=0.002(p=0.99)$. 
Interval from RT

The correlation of adventitial contrast intensity difference (irradiated - unirradiated) to interval from RT is shown in figure 3 (attenuation-corrected images). This shows no correlation between the interval from RT and the difference in contrast intensity between the two sides of the neck. This suggests that contrast intensity does not increase steadily over time. Rather, it is more likely that this increase following RT occurs at a certain time point and then remains stable over time. As all patients had been treated at least 2 years previously, it is probable that this process occurs prior to 2 years following RT.

\section{DISCUSSION}

This study has demonstrated a significant difference in contrast intensity between irradiated and unirradiated carotid arteries with novel software that utilizes an attenuation correction and image normalization method. This is strengthened by the fact that no significant difference was seen between the two sides of the neck on subtraction images (i.e. before the infusion of contrast). Attenuation artefacts in CEUS images, together with other factors, can make reliable quantification difficult and lead to diagnostic uncertainty. This is the first study, as far as we are aware, to utilize attenuation and normalisation techniques for CEUS quantification in irradiated carotid arteries. In addition, the use of internal controls within the same patient makes this an attractive model for investigating increased contrast intensity following RT.

The intensity per pixel parameter derived in this study has not been utilized before and requires validation in a longitudinal study and correlation to histological samples. We believe, however, that the average intensity per pixel will be more representative of changes occurring in the adventitia and less likely to over- or underestimate contrast intensity in the adventitia. 
There have been studies investigating adventitial vv proliferation in other settings. Sampson et al (21) investigated carotid vv ratio (semi-automated software was used to derive the video intensity in the adventitia over the video intensity in the lumen) in diabetic and nondiabetic patients. They demonstrated that $\mathrm{vv}$ ratio was unrelated to CIMT, but both parameters were independently associated with diabetes. This is in keeping with the findings from our study, which showed no correlation between contrast intensity difference between the two sides of the neck and CIMT. It is therefore possible that vv proliferation may be an independent risk factor for atherosclerosis but this would require further investigation in longitudinal studies.

It is important to note, however, that there are considerable methodological differences in that our study considered the intensity per pixel rather than a $2 \mathrm{~mm}$ area of adventitia. Both studies considered 2-dimensional views of the arterial vessel with selection of a small region of interest. Therefore, measurements obtained may not be representative of the entire vessel. What is clear, however, is that both studies demonstrate increased contrast uptake in high-risk groups and our study has the advantage of using matched internal controls. In addition, we have corrected for attenuation artefacts with our quantification software, which were not accounted for in the other study. In CEUS, pseudo-enhancement artefact originating from tissues could be present due to ultrasound non-linear propagation $(22,23)$. To rule out the possibility that different tissue echogenicity caused the difference in CEUS signals in our study, non-contrast video loops of arteries on both sides of the neck were obtained prior to contrast infusion. There was no significant difference in adventitial intensity between the two sides of the neck prior to contrast and, therefore, we are confident that differences in intensity between the two sides following contrast infusion were likely to have been due to the contrast uptake in the adventitial vv.

Schinkel et al (24) used CEUS to assess carotid wall vascularization in a cohort of patients with large vessel vasculitis (Takayasu or giant cell arteritis) using a previously published 
qualitative grading method (25). They reported carotid wall vascularization in $64 \%$ of carotid arteries (29\% of which were graded as severe wall vascularization). The authors did note that previously reported pseudo-enhancement artefact $(26,27)$ may over-estimate contrast activity in the far wall. The study did demonstrate, however, increased contrast intensity in a vasculitic process, supporting the premise that adventitial $v v$ proliferation is part of an inflammatory process that may be detected by CEUS.

What is still uncertain is whether the increased contrast intensity is due to proliferation of vv or some other process related to RT-induced damage e.g. perivascular and/or intravascular fibrosis. Correlation of CEUS features with histological features would provide further clarification. The contrast intensity difference does not increase with interval from RT and this suggests that, once the intensity increases due to whatever process is occurring, it remains relatively stable over time.

There was no correlation between the contrast intensity difference and the difference in the distal CCA CIMT or the QSTROKE score. Therefore, further prospective work is required to determine if this imaging biomarker is truly an early marker of radiation-induced atherosclerosis. Prospective data using this model of matched internal controls would provide useful information about the timing of adventitial vv proliferation after RT and its correlation to CIMT at different time points. It is also possible that this marker is an independent biomarker and may correlate to the final end-point of stroke or TIA but longterm data are required to clarify this. An early imaging biomarker of radiation-induced atherosclerosis would allow for detection of preclinical changes in the carotid artery and risk stratification of patients for monitoring and risk reduction therapies, as well as the assessment of response to antiatherosclerotic therapies. This technique may also be useful to assess the success of carotid-sparing RT techniques. 
Ultimately, longitudinal data are required to determine if CEUS may be useful as an imaging biomarker for radiation-induced damage. The use of targeted microbubbles to activated leukocytes (28) may be more useful in identifying changes in both plaques and the adventitia after RT. In addition, the use of 3-D ultrasound techniques may provide a more representative picture of the processes occurring in the arterial wall and plaques.

\section{CONCLUSION}

We have demonstrated that contrast intensity is significantly increased in irradiated carotid arteries and may be a useful imaging biomarker for radiation-induced atherosclerosis. Our study supports previous data that this biomarker may be independently associated with vascular damage and is not associated to CIMT. Studies in other high-risk groups have shown increased contrast uptake in keeping with an inflammatory process. Refinement of CEUS techniques to account for attenuation artifact and pseudoenhancement in the far wall of the carotid artery is still required but we have gone some way in addressing these issues.

\section{Acknowledgements}

This work was undertaken in The Royal Marsden NHS Foundation Trust which received a proportion of its funding from the NHS Executive; the views expressed in this publication are those of the authors and not necessarily those of the NHS Executive. This work was supported by the Cancer Research UK Programme Grants C7224/A13407. The authors also acknowledge the support of the National Institute for Health Research Royal Marsden and Institute of Cancer Research Biomedical Research Centre.

\section{Funding}

None

\section{Conflict of Interest Notification}

We declare no conflicts of interest 


\section{REFERENCES}

1. Kwon HM, Sangiorgi G, Ritman EL, McKenna C, Holmes DR, Jr., Schwartz RS, et al. Enhanced coronary vasa vasorum neovascularization in experimental hypercholesterolemia. J Clin Invest. 1998;101(8):1551-6. PMCID: 508734.

2. Herrmann J, Lerman LO, Rodriguez-Porcel M, Holmes DR, Jr., Richardson DM, Ritman EL, et al. Coronary vasa vasorum neovascularization precedes epicardial endothelial dysfunction in experimental hypercholesterolemia. Cardiovasc Res. 2001;51(4):762-6.

3. Virmani R, Burke AP, Farb A, Kolodgie FD. Pathology of the vulnerable plaque. J Am Coll Cardiol. 2006;47(8 Suppl):C13-8.

4. Feinstein SB. Contrast ultrasound imaging of the carotid artery vasa vasorum and atherosclerotic plaque neovascularization. J Am Coll Cardiol. 2006;48(2):236-43.

5. Macioch JE, Katsamakis CD, Robin J, Liebson PR, Meyer PM, Geohas C, et al. Effect of contrast enhancement on measurement of carotid artery intimal medial thickness. Vasc Med. 2004;9(1):7-12.

6. Feinstein SB. The powerful microbubble: from bench to bedside, from intravascular indicator to therapeutic delivery system, and beyond. Am J Physiol Heart Circ Physiol. $2004 ; 287(2): H 450-7$.

7. Folkman J. The vascularization of tumors. Sci Am. 1976;234(5):58-64, 70-3.

8. Barger AC, Beeuwkes R, 3rd, Lainey LL, Silverman KJ. Hypothesis: vasa vasorum and neovascularization of human coronary arteries. A possible role in the pathophysiology of atherosclerosis. N Engl J Med. 1984;310(3):175-7.

9. Granada JF, Feinstein SB. Imaging of the vasa vasorum. Nat Clin Pract Cardiovasc Med. 2008;5 Suppl 2:S18-25.

10. Magnoni M, Coli S, Marrocco-Trischitta MM, Melisurgo G, De Dominicis D, Cianflone D, et al. Contrast-enhanced ultrasound imaging of periadventitial vasa vasorum in human carotid arteries. Eur J Echocardiogr. 2009;10(2):260-4. 
11. Coli S, Magnoni M, Sangiorgi G, Marrocco-Trischitta MM, Melisurgo G, Mauriello A, et al. Contrast-enhanced ultrasound imaging of intraplaque neovascularization in carotid arteries: correlation with histology and plaque echogenicity. $J$ Am Coll Cardiol. 2008;52(3):223-30.

12. Shah F, Balan P, Weinberg M, Reddy V, Neems R, Feinstein M, et al. Contrastenhanced ultrasound imaging of atherosclerotic carotid plaque neovascularization: a new surrogate marker of atherosclerosis? Vasc Med. 2007;12(4):291-7.

13. Staub D, Patel MB, Tibrewala A, Ludden D, Johnson M, Espinosa P, et al. Vasa vasorum and plaque neovascularization on contrast-enhanced carotid ultrasound imaging correlates with cardiovascular disease and past cardiovascular events. Stroke. 2010;41(1):41-7.

14. Tang MX, Mari JM, Wells PN, Eckersley RJ. Attenuation correction in ultrasound contrast agent imaging: elementary theory and preliminary experimental evaluation. Ultrasound Med Biol. 2008;34(12):1998-2008.

15. Tang MX, Mulvana H, Gauthier T, Lim AK, Cosgrove DO, Eckersley RJ, et al. Quantitative contrast-enhanced ultrasound imaging: a review of sources of variability. Interface Focus. 2011;1(4):520-39. PMCID: 3262271.

16. Mari J, Hibbs K, Stride E, Eckersley R, Tang M. An approximate nonlinear model for time gain compensation of amplitude modulated images of ultrasound contrast agent perfusion. IEEE Trans Ultrason Ferroelectr Freq Control. 2010;57(4):818-29.

17. Mule S, De Cesare A, Lucidarme O, Frouin F, Herment A. Regularized estimation of contrast agent attenuation to improve the imaging of microbubbles in small animal studies. Ultrasound Med Biol. 2008;34(6):938-48.

18. Cheung WK, Gujral DM, Shah BN, Chahal NS, Bhattacharyya S, Cosgrove DO, et al. Attenuation Correction and Normalisation for Quantification of Contrast Enhancement in Ultrasound Images of Carotid Arteries. Ultrasound Med Biol. 2015;41(7):1876-83. 
19. Golemati S, Sassano A, Lever MJ, Bharath AA, Dhanjil S, Nicolaides AN. Carotid artery wall motion estimated from B-mode ultrasound using region tracking and block matching. Ultrasound Med Biol. 2003;29(3):387-99.

20. Greenland P, Alpert JS, Beller GA, Benjamin EJ, Budoff MJ, Fayad ZA, et al. 2010 ACCF/AHA guideline for assessment of cardiovascular risk in asymptomatic adults: a report of the American College of Cardiology Foundation/American Heart Association Task Force on Practice Guidelines. J Am Coll Cardiol. 2010;56(25):e50-103.

21. Sampson UK, Harrell FE, Jr., Fazio S, Nwosu S, Mercaldo N, Mensah GA, et al. Carotid Adventitial Vasa Vasorum and Intima-Media Thickness in a Primary Prevention Population. Echocardiography. 2014.

22. Tang MX, Eckersley RJ. Nonlinear propagation of ultrasound through microbubble contrast agents and implications for imaging. IEEE Trans Ultrason Ferroelectr Freq Control. 2006;53(12):2406-15.

23. Tang MX, Kamiyama N, Eckersley RJ. Effects of nonlinear propagation in ultrasound contrast agent imaging. Ultrasound Med Biol. 2010;36(3):459-66.

24. Schinkel AF, van den Oord SC, van der Steen AF, van Laar JA, Sijbrands EJ. Utility of contrast-enhanced ultrasound for the assessment of the carotid artery wall in patients with Takayasu or giant cell arteritis. Eur Heart J Cardiovasc Imaging. 2014;15(5):541-6.

25. Staub D, Partovi S, Schinkel AF, Coll B, Uthoff H, Aschwanden M, et al. Correlation of carotid artery atherosclerotic lesion echogenicity and severity at standard US with intraplaque neovascularization detected at contrast-enhanced US. Radiology. $2011 ; 258(2): 618-26$.

26. Thapar A, Shalhoub J, Averkiou M, Mannaris C, Davies AH, Leen EL. Dosedependent artifact in the far wall of the carotid artery at dynamic contrast-enhanced US. Radiology. 2012;262(2):672-9.

27. ten Kate GL, Renaud GG, Akkus Z, van den Oord SC, ten Cate FJ, Shamdasani V, et al. Far-wall pseudoenhancement during contrast-enhanced ultrasound of the carotid 
arteries: clinical description and in vitro reproduction. Ultrasound Med Biol. 2012;38(4):593600.

28. Lindner JR, Song J, Xu F, Klibanov AL, Singbartl K, Ley K, et al. Noninvasive ultrasound imaging of inflammation using microbubbles targeted to activated leukocytes. Circulation. 2000;102(22):2745-50. 


\section{Figures and Tables}

Figure 1. Contrast adventitial intensity in irradiated (red) versus unirradiated (green) arteries a) before attenuation correction and b) after attenuation correction ( $p$ significant if $<0.05)$. The horizontal black lines represent the mean and SD.

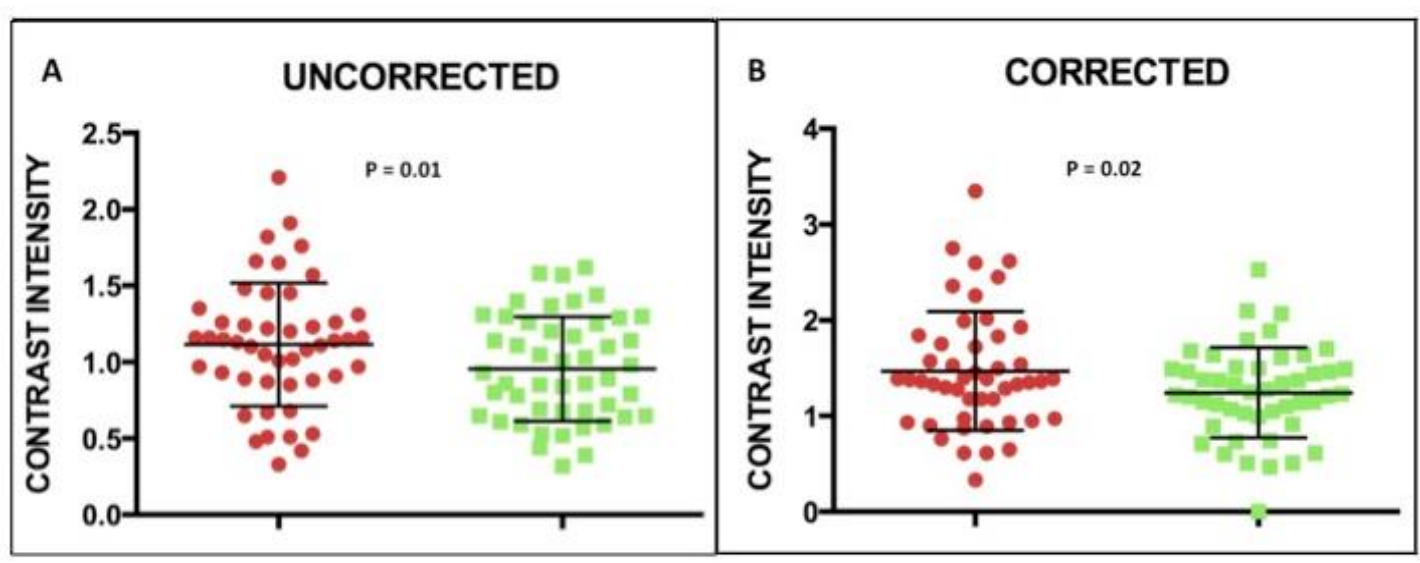


Figure 2. Correlation of distal CCA CIMT difference (irradiated - unirradiated) and contrast intensity difference (irradiated - unirradiated) a) before and b) after attenuation correction ( $p$ significant if <0.005)
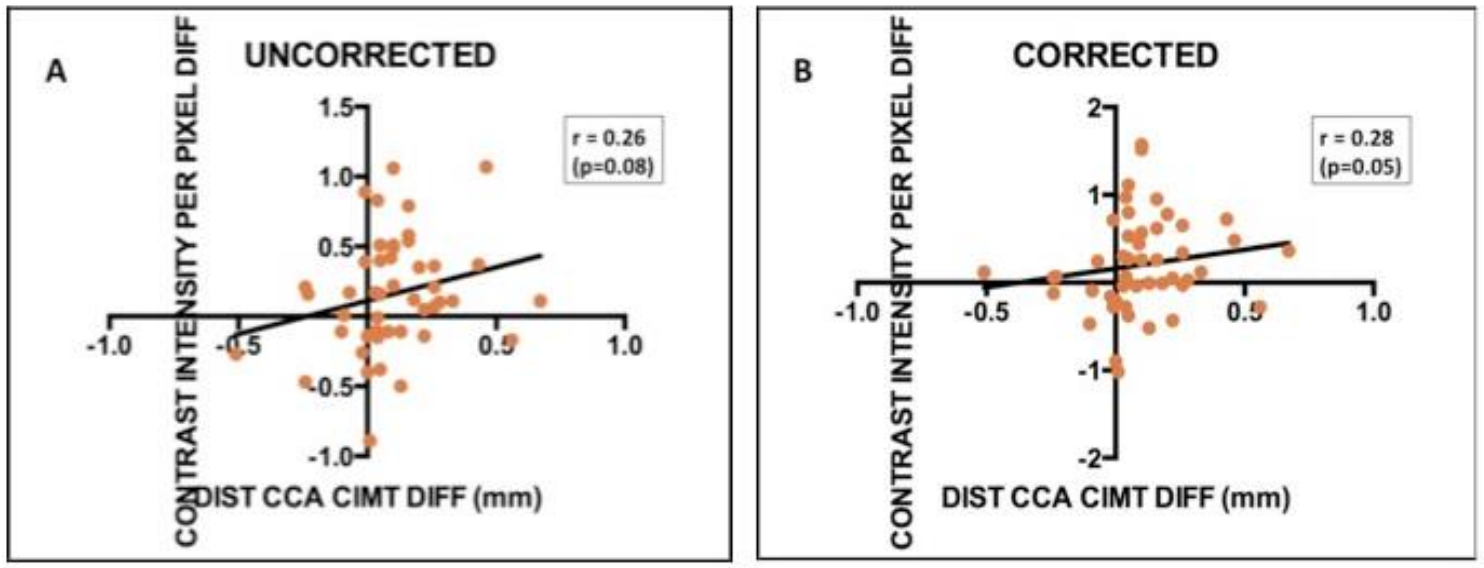
Figure 3. Correlation of contrast intensity difference (irradiated - unirradiated) and interval from RT ( $p$ significant if $<0.005)$

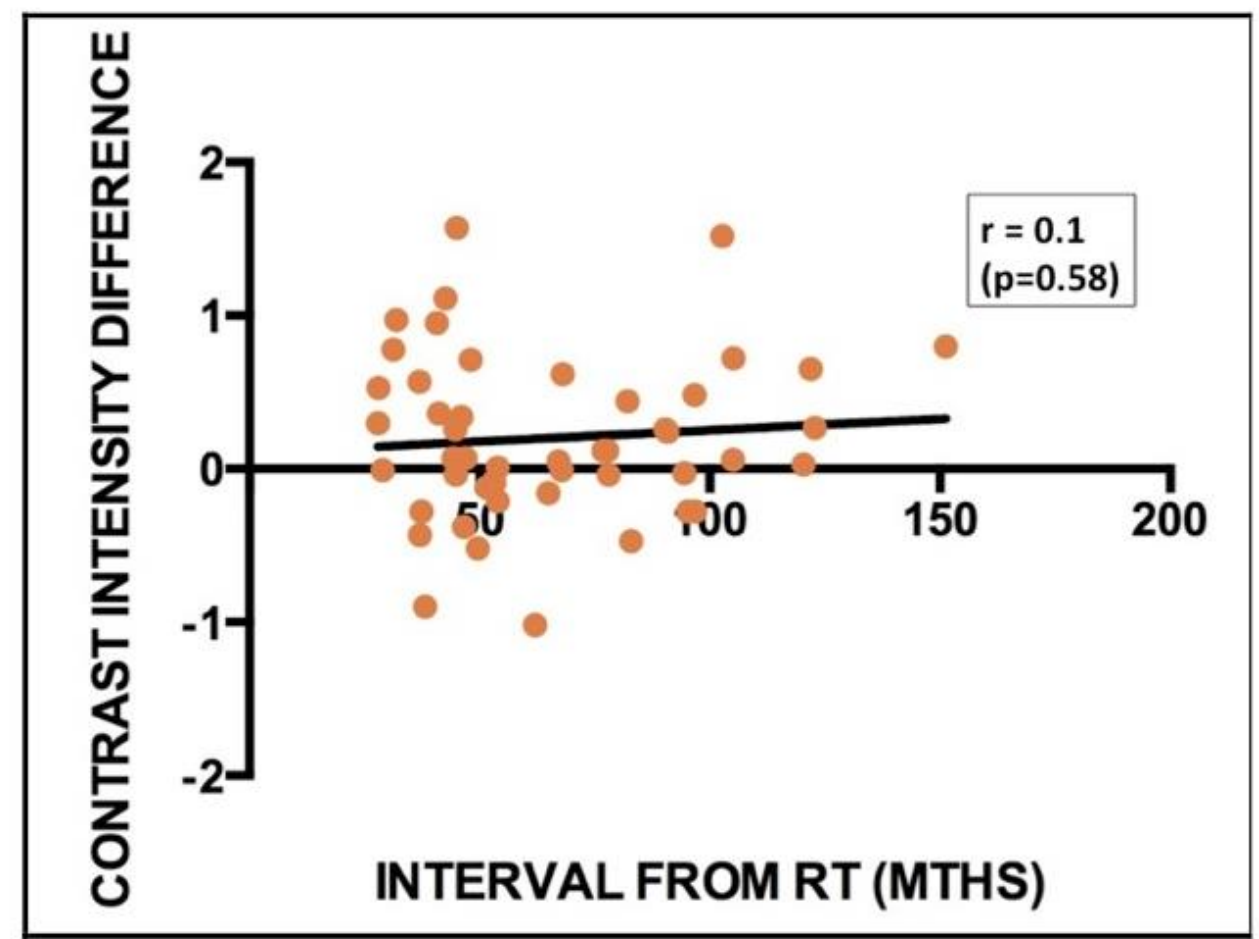


Table 1. Patient demographics

\begin{tabular}{|c|c|c|}
\hline Demographic ch & acteristics/risk factors & Number (\%) ( $\mathrm{N}=48)$ \\
\hline Female & & $15(31 \%)$ \\
\hline Age in years, $m$ & ian (interquartile range) & $59.2(49.2-64.2)$ \\
\hline Histology & Squamous & $38(79 \%)$ \\
\hline & Non-squamous & $10(21 \%)$ \\
\hline Tumour Grade & Well Differentiated & $2(4 \%)$ \\
\hline & Moderate Differentiated & $8(17 \%)$ \\
\hline & Poorly Differentiated & $25(52 \%)$ \\
\hline & Unknown & $13(27 \%)$ \\
\hline Tumour Stage & 0 & $7(15 \%)$ \\
\hline & 1 & $20(42 \%)$ \\
\hline & 2 & $17(35 \%)$ \\
\hline & 3 & $1(2 \%)$ \\
\hline & 4 & $3(6 \%)$ \\
\hline Nodal Status & 0 & $11(23 \%)$ \\
\hline & 1 & $10(21 \%)$ \\
\hline & 2 & $27(56 \%)$ \\
\hline Neck Dissection & & $31(65 \%)$ \\
\hline Induction Chem & herapy & $7(15 \%)$ \\
\hline Concomitant Ch & notherapy & $22(46 \%)$ \\
\hline$R T$ dose to nec & 50 Gray & $19(40 \%)$ \\
\hline (Gy) & 60 Gray & $28(58 \%)$ \\
\hline & 63 Gray & $1(2 \%)$ \\
\hline Interval since ra & otherapy (months) & $59.4(41-88.7)$ \\
\hline Risk Factors & Diabetes & $4(8 \%)$ \\
\hline & Hypertension & $12(28 \%)$ \\
\hline & Dyslipidaemia & $11(23 \%)$ \\
\hline & Smoker & $25(52 \%)$ \\
\hline Body Mass Inde & $\left(\mathrm{kg} / \mathrm{m}^{2}\right)$ (median (IQR)) & $26.5(23.2-28.4)$ \\
\hline Medication & Aspirin & $12(28 \%)$ \\
\hline & ACE Inhibitors & $4(8 \%)$ \\
\hline & $\begin{array}{l}\text { 5-HMG } \quad \text { Co-reductase } \\
\text { Inhibitor }\end{array}$ & $13(27 \%)$ \\
\hline Total Cholesterc & mmol/L) & $5.1(4.6-5.8)$ \\
\hline Low Density Lip & oroteins (mmol/L) & $3.3(2.6-3.7)$ \\
\hline High Density Lik & proteins (mmol/L) & $1.4(1.2-1.7)$ \\
\hline
\end{tabular}


Table 2. Effect of surgery and chemotherapy on adventitial contrast intensity before attenuation correction (uncorr) and after correction (corr) ( $p$ is significant if $<0.025$ )

\begin{tabular}{l|l|l|l|l|l}
\hline \multicolumn{7}{c}{ Contrast intensity per pixel } \\
\hline \multirow{2}{*}{} & & $\begin{array}{l}\text { Irradiated } \\
\text { Median (IQR) }\end{array}$ & P value & $\begin{array}{l}\text { Unirradiated } \\
\text { Median (IQR) }\end{array}$ & P value \\
\hline UNCORR & Neck dissection & $1.1(0.83-1.4)$ & 0.80 & $1.1(0.61-1.6)$ & 0.70 \\
& No neck dissection & $1.1(0.95-1.3)$ & & $0.86(0.71-1.1)$ & \\
\hline CORR & Neck dissection & $1.4(0.85-1.8)$ & 0.94 & $1.3(0.81-1.5)$ & 0.14 \\
& No neck dissection & $1.3(1.1-1.6)$ & & $0.97(0.85-1.2)$ & \\
\hline UNCORR & Chemotherapy & $1.1(0.92-1.3)$ & 0.75 & $0.81(0.63-1.2)$ & 0.17 \\
& No chemotherapy & $1.1(0.68-.3)$ & & $1.1(0.77-1.3)$ & \\
\hline CORR & Chemotherapy & $1.5(1.1-1.7)$ & 0.42 & $0.91(0.79-1.5)$ & 0.16 \\
& No chemotherapy & $1.2(0.95-1.7)$ & & $1.3(0.96-1.5)$ & \\
\hline
\end{tabular}

